\title{
PET-CT successfully identifies ccRCC
}

A new phase III study (REDECT) has shown for the first time that highly malignant clear cell renal cell carcinoma (ccRCC) can be identified using PETCT imaging with high sensitivity and specificity. This finding could inform treatment options in patients who are likely to develop metastatic disease, as well as minimize the need for surgical biopsy in patients who are unfit for such a procedure. ccRCC is associated with poor prognosis owing to its high metastatic potential. "This is the first prospective multicentre study that demonstrates the ability of PET-CT to identify an aggressive cancer phenotype (ccRCC)," explains lead investigator Chaitanya Divgi, who is based at Columbia University in New York.

Approximately $95 \%$ of cRCC cells express the cell-surface antigen carbonic anhydrase IX, an expression pattern leveraged by Divgi and his colleagues to develop a chimeric antibody (girentuximab) against the protein. They conjugated this antibody with iodine-124 $\left.{ }^{124} \mathrm{I}\right)$, a positron-emitting radionuclide, for use with PET-CT.

226 patients were enrolled in the study across 14 centres in the USA. Of the 202 patients who underwent surgery, $42 \mathrm{had}$ multiple renal lesions. ${ }^{124}$ I-girentuximab was well tolerated by patients, with the vast majority of adverse events being grade 1 or 2-the most common effects were headache, diarrhoea and dizziness.

All images were centrally interpreted by three blinded readers and tumour histology was determined by a blinded central pathologist. Compared with contrast-enhanced CT, the PET-CT method outperformed in terms of average sensitivity $(86.2 \%$ versus $75.5 \%, P=0.023)$ and specificity $(85.9 \%$ versus $46.8 \%$, $P=0.005)$ for ccRCC. Furthermore, the sensitivity was higher for lesions between $2 \mathrm{~cm}$ and $4 \mathrm{~cm}$ in size (89.4\%). The researchers also calculated positive and negative predictive values as secondary efficacy variables, finding the technique based on ${ }^{124}$ I-girentuximab performed better than contrast-enhanced CT.

Importantly, both inter-reader and intrareader agreement were high for PET-CT compared with contrastenhanced CT. Indeed, the percentage agreement between two evaluations of images from a randomly selected subset of patients ranged from $87 \%$ to $100 \%$ for PET-CT, which was considerably better than the range for contrast-enhanced CT (73.7-91.3\%). These results speak to the robustness of ${ }^{124} \mathrm{I}$-girentuximab in detecting ccRCC, which, as Divgi explains, "will be tremendously useful in the assessment of renal masses, potentially avoiding unnecessary surgery as well as informing the nature of any surgical procedure." Although PET-CT is not inexpensive, its benefit might exceed the costs and morbidities associated with surgery and even biopsy.

Indeed, in some patients, this noninvasive imaging procedure might be used to replace or guide biopsy. Such

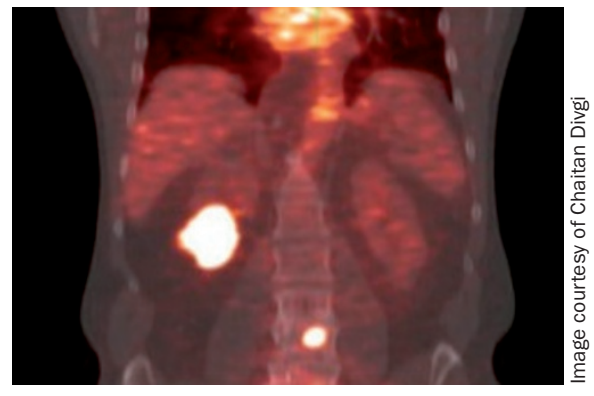

a tool is of particular importance for patients who are unfit for surgery, elderly or who have comorbidities that preclude surgery. Furthermore, using the PET-CT method for detecting small renal masses is particularly attractive in light of the growing body of evidence that these patients might benefit more from active surveillance than radical surgery. In this regard, four lesions $\leq 1 \mathrm{~cm}$ were detected and correctly diagnosed in the REDECT trail, which bodes well for these patients.

In addition to being a specific noninvasive imaging biomarker for malignancy, ${ }^{124}$ I-girentuximab might be useful as, Divgi continues, "a pharmacodynamic biomarker to establish the utility of systemic therapies in the treatment of metastatic ccRCC." Additional studies to evaluate the agent as a pharmacodynamic marker in ccRCC are underway.

Mina Razzak
Original article Divgi, C. R. et al. Positron emission tomography/computed tomography identification of clear cell renal cell carcinoma: results from the REDECT trial. J. Clin. Oncol. doi:10.1200/JC0.2011.41.2445 